\title{
Non-Alcoholic Fatty Liver Disease in Primary Hypothyroidism
}

Ahmed NU1, Kabir $\mathrm{MA}^{2}$, Kalam F3, Akter S4

DOI: https:/ / doi.org/ 10.3329/ jafmc.v15i2.50838

\section{Abstract}

Introduction: Non-alcoholic fatty liver disease (NAFLD) is the most common chronic liver condition world-wide. Hypothyroidism is also a common disorder affecting general population especially in women.

Objectives: To determine the association between primary hypothyroidism and NAFLD.

Materials and Method: This is a cross-sectional descriptive type of observational study where 100 cases of primary hypothyroidism in age group 15-75 years of both sexes were selected from February 2018 to January 2019 in CMH Momenshahi having higher level of Thyroid stimulating hormone(TSH). In all cases ultra-sonogram of hepatobiliary system was done by efficient sonologist who was blind about clinical scenario of the patients.

Results: Mean age of patients 29 \pm SD7.57. Among 100 cases $56(56 \%)$ having NAFLD among them 95\% were female and 5\% were male.

Conclusion: NAFLD was significantly correlated with primary hypothyroidism.

Key-words: Primary Hypothyroidism, Non-alcoholic fatty liver disease, Cholesterol, Triglycerides, high density lipoprotein, low density lipoprotein.

\section{Introduction}

Nonalcoholic fatty liver disease (NAFLD) is the most common chronic liver condition worldwide ${ }^{1}$. It is defined as hepatic steatosis by imaging or histology but without secondary causes of hepatic fat accumulation, such as significant alcohol consumption and long term use of steatogenic medication ${ }^{2}$. In this world, the prevalence of NAFLD ${ }^{3,4}$ diagnosed by imaging in general population is about $25 \%$ and in Bangladesh $34.34 \%$. The growing pattern of NAFLD prevalence is generally attributed to global increase in the prevalence of obesity and other metabolic risk factors ${ }^{5}$. Reduced production of thyroid hormone is the central feature of the clinical state termed hypothyroidism ${ }^{6,7}$. Thyroid disorders are common in the general population with hypothyroidism being the predominant disorder in the adult population,8.9.

Thyroid hormones play an important role in regulating body weight, lipid metabolism and insulin resistance. Low thyroid hormone levels are related with hypo-metabolism categorized by decreased weight gain, resulting energy expenditure, reduced lipolysis, increased cholesterol levels and reduced gluconeogenesis ${ }^{10}$.
Therefore, thyroid hormones may have a close relationship with the pathogenesis of NAFLD ${ }^{11}$. However, the association of NAFLD with thyroid function or hypothyroidism remain controversial in adults ${ }^{12,13}$. An expanding number of illnesses have been accounted for to be to NAFLD; for example, cancer, cardiovascular ailment, type 2 diabetes, and chronic kidney ailment14,15. This study is an effort to assess the association between primary hypothyroidism and NAFLD

\section{Materials and Method}

This is a cross sectional descriptive type of observational study of thyroid patient where a total of 100 were studied during February 2018 to January 2019. Patients of both sexes, age limit from 13 to 70 years and suspected case of hypothyroidism were included. Patients with hepatitis or liver cirrhosis or with hepatotoxic drug intake or associated any debilitating illness and pregnant women were excluded. All those patients were seen in medical outdoor department of Combined Military Hospital (CMH), Momenshahi. A detailed history and clinical examination were carried out in a predesigned case record form. Under aseptic precaution morning sample of venous blood was taken from patients of hypothyroidism after 12 hours overnight fasting with disposable syringe and needle. Serum FT-4, TSH were measured by chemilunescence immunoassay by Cobas C411. Patients with TSH level $6 \mu \mathrm{IU} / \mathrm{ml}$ and above were considered to be having hypothyroidism. Abdominal ultra-sonogram scanning was performed in all cases by a skilled sonologist by $7.5 \mathrm{MHz}$ linear transducer who was blind to all clinical and laboratory data of patient.

\section{Results}

Out of 100 patients $56 \%$ of the patients are from age group 26-35 years, 26\% from $36-45$ years, 14\% from 15-25 years, 3\% from $46-55$ years and $1 \%$ from $66-75$ years. Mean age of patient $29 \pm 7.57$ years. Most of the patients are from age group 26-35 years (Table-I). Fig-1 Bar chart showing clinical presentation of hypothyroidism patients. Most of the patients (18\%) presented with vague symptoms like general weakness, $15 \%$ with menstrual irregularity, $14 \%$ with weight gain. Other presentations are thyroid swelling (11\%), subfertility $(10 \%)$, leg swelling $(8 \%)$, body ache (8\%), incidental finding $(5 \%)$, abortion (4\%), cold intolerance (2\%), decreased libido (2\%), alopecia $(1 \%)$, whitish spots (1\%) and 1\% recurrent syncope (Figure-1). About $55 \%$ patients having fatty liver and $44 \%$ having no fatty liver (Figure2). Barr chart showing mean level of cholesterol, TG, HDL and LDL $195.14 \pm 44.57,164.49 \pm 83.87,40 \pm 3.91$ and $122 \pm 41.72$ respectively (in $\mathrm{mg} / \mathrm{dL}$ ) (Fig-3).

1. Lt Col Nasir Uddin Ahmed, MBBS, FCPS, Classified Specialist in Medicine, CMH, Dhaka (E-mail: nasir_amc@yahoo.com) 2. Brig Gen Md Anwarul Kabir, MBBS, FCPS, DEM, Adviser Specialist in CMH, Dhaka 3. Lt Col Farzana Kalam, MBBS, DA, MCPS, FCPS, Classified Specialist in Anaesthesiology, CMH, Dhaka 4. Maj Shaheda Akter, MBBS, MCPS, Graded Specialist in Radiology and Imaging, CMH, Dhaka. 
Table-l: Distribution of age and sex of patients $(n=100)$

\begin{tabular}{|l|c|c|c|}
\hline \multicolumn{2}{|c|}{ Characteristics } & Frequency & Percentage \\
\hline \multirow{4}{*}{$\begin{array}{l}\text { Age in } \\
\text { years }\end{array}$} & $15-25$ & 14 & 14 \\
\cline { 2 - 4 } & $26-35$ & 56 & 56 \\
\cline { 2 - 4 } & $36-45$ & 26 & 26 \\
\cline { 2 - 4 } & $46-55$ & 3 & 3 \\
\cline { 2 - 4 } & $56-65$ & 0 & 0 \\
\cline { 2 - 4 } & $66-75$ & 1 & 1 \\
\cline { 2 - 4 } & Mean \pm SD & \multicolumn{2}{|c|}{$29 \pm 7.57$} \\
\hline \multirow{4}{*}{ Sex } & Male & 5 & 5 \\
\cline { 2 - 4 } & Female & 95 & 95 \\
\hline
\end{tabular}

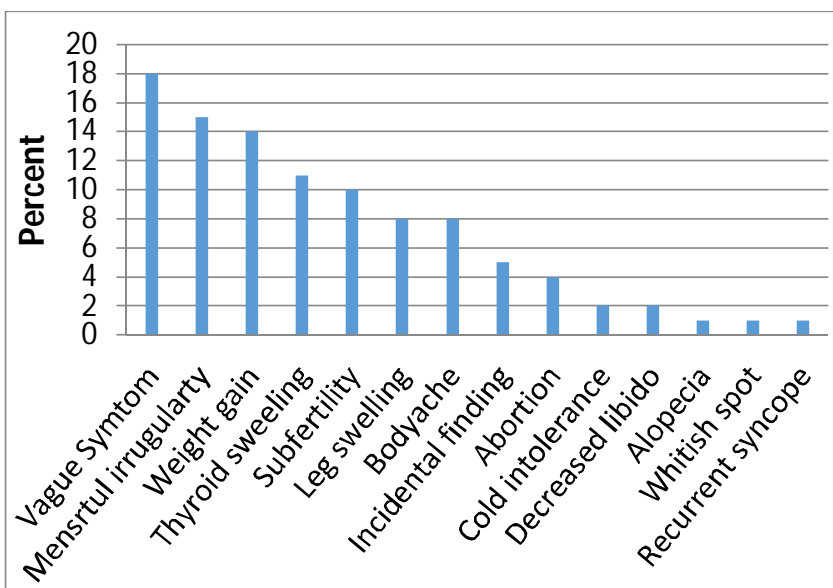

Clinical Presentation

Figure-1: Clinical presentation of primary hypothyroidism

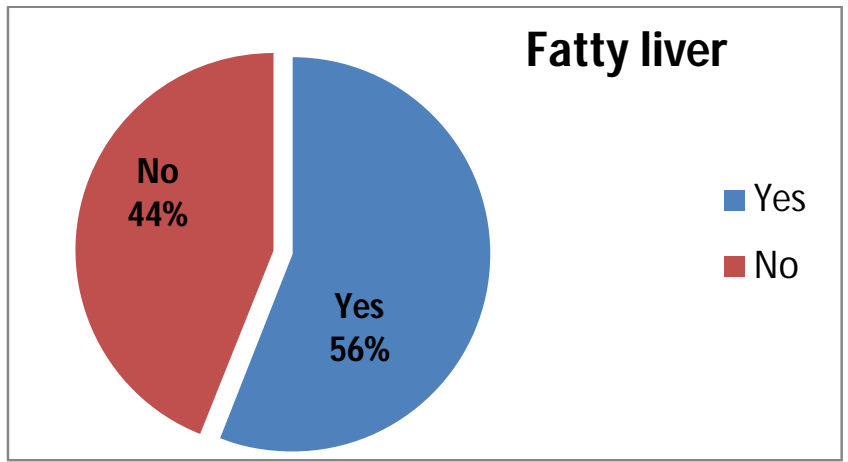

Figure-2: Fatty liver status

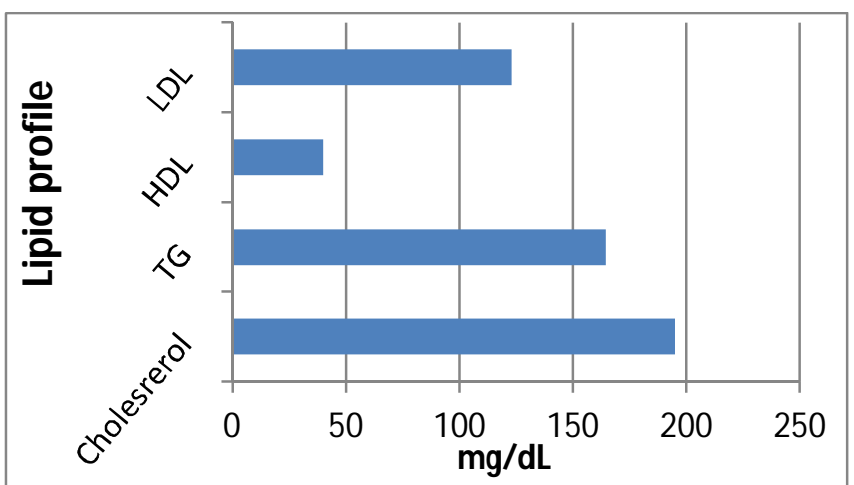

Figure-3: Lipid profile of patients.

\section{Discussion}

In this study $95 \%$ are female and $5 \%$ are male which seems to be higher than the prevalence of hypothyroidism in female. As we have conducted this study in Combined Military Hospital where male are recruited after thorough medical examination but their ladies are not. So there might be more chance of disease in their ladies than male. NAFLD is supposed to be a hepatic feature of metabolic syndrome and insulin resistance ${ }^{16,17}$. Hypothyroidism has been reported to be associated with obesity and metabolic syndrome ${ }^{18,19}$. All these factors are responsible for NAFLD in hypothyroidism. This study found that the prevalence of NAFLD was $56 \%$ which seemed to be higher than the general prevalence in Asia (27.4\%; $95 \%$ confidence interval $23.3 \%-31.9 \%)$. It is also higher than the prevalence in general population in Bangladesh (34.34\%) $)^{4}$. In this study, it is found significant ( $p$ value $<0.005$ with $z$ value 4.64 )

This finding was similar to study done by Yiting $L$ et al 20 which found NAFLD was $35.98 \%$. It is little bit lower than this study which can be explained by geographical variation and more female patients in this study group. This study was in agreement with studies done by Arafat $\mathrm{K}$ et $\mathrm{a}^{21}$ and Weiwei $\mathrm{H}$ et $\mathrm{a}^{22}$. In both the studies found that hypothyroidism was independently correlated with NAFLD. On the contrary results of some studies by Mazo et al ${ }^{23}$ Eshraghian et al24 failed to demonstrate association between markers of thyroid dysfunction and presence of NAFLD. These dissimilarities may be explained that their studies were done in treated cases of hypothyroidism but in this study we have taken sample both from new and also treated old cases.

NAFLD defines a spectrum of histological abnormalities from simple fatty liver to nonalcoholic steohepatitis, in a person consuming no alcohol25. Thyroid hormones can stimulate expression of uncoupling proteins in the mitochondria of fat and skeletal muscle, modulate adrenergic receptors numbers by enhancing responsiveness of catecholamines, and thus control metabolic and energy homeostasis ${ }^{18}$. Leptin is considered as one of the explanations of this association because it is found to be increased in patients with hypothyroidism and it is also found to be higher in NAFLD as it can promote hepatic insulin resistance and be involved in hepatic fibro genesis $23-25$.

\section{Conclusion}

The results of the present study confirm the correlation between hypothyroidism and nonalcoholic fatty liver disease and which is statistically significant. An increased TSH is an independent risk factor for NAFLD.

\section{References}

1. Nascimbeni $F$, Pais $R$, Bellentani $S$ et al. NAFLD in clinical practice to answer from guidelines. J Hepatol 2013; 59:859-71.

2. Chalasani N, Younossi Z, Lavine JE et al. The diagnosis and management of non-alcoholic fatty liver disease: Practice guidance from American Association for the study of liver disease. Hepatology 2018; 67:328-57

3. Younossi ZM, Koenig $A B$, Abdelatif $D$ et al. Global epidemiology of non-alcoholic fatty liver disease: Meta analytic assessment of prevalence, incidence and outcomes. Hepatology 2016; 64:73-84. 
4. Shahinul A, Muhammad A, Golam A et al. Prevalence of fatty liver in Bangladesh: A nation-wide population based study. Hepatol Int 2017; 11 (suppl 1):S1-S1093.

5. Day CP. Non-alcoholic fatty liver disease: A massive problem. Clin med 2011; 11:176-8.

6. Vaidya $\mathrm{B}$, Pearce $\mathrm{SH}$. Management of hypothyroidism in adults. MBJ 2008; 337:801.

7. Almandoz JP, Gharib H. Hypothyroidism: Etiology, diagnosis, and management. Med clin North Am 2012; 96:203-21.

8. Rizos CV, Elisaf MS, Liberopulos EN. Effects of thyroid dysfunction on lipid profile. Open Cardiovasc Med J 2001; 5:76-84.

9. Wu P. Thyroid disease and diabetes: Clin Diabetes 2000; 18:38-40.

10. Pucci E, Chiovato L, Pinchera A. Thyroid and lipid metabolism. Trends Endocrinol Metab 2014; 25:538-5

11. Eshraghian A, Jahromi AH. Non-alcoholic fatty liver disease and thyroid dysfunction: A systemic review. World J Gastroenterol 2014; 20:8102-9.

12. Moustafa AHA, Ali EMM, Mohamed TM et al. Oxidative stress and thyroid hormones in patients with liver disease. Eur J Intern Med 2009; 20:703-8.

13. Pagadala MR, Zein CO, Dasarathy $S$ et al. Prevalence of hypothyroidism in non-alcoholic fatty liver disease. Dig Dis Sci 2012; 57:528-34.

14. Motamed N, Rabiee B, Poustchi $\mathrm{H}$ et al. Non- alcoholic fatty liver disease and 10 years risk of cardiovascular disease. Clin Res Hepatol Gastroenterol 2017; 41(1):31-8.

15. Adams $\mathrm{LA}$, Anstee $\mathrm{QM}$, Tilg $\mathrm{H}$ et al. Non-alcoholiuc fatty liver disease and its relationship with cardiovascular disease and other extra hepatic disease. Gut 2017; 66(6):1138-53.
16. Yilmaz Y. NAFLD in the absence of metabolic syndrome: Different epidemiology, pathogenetic mechanisms, risk factors for disease progression? Semi Liver Dis 2012; 32:14-21.

17. Collantes RS, Ong JP, Younossi ZM. The metabolic syndrome and non-alcoholic fatty liver disease. Panminerva Med 2006; 48:41-8.

18. Pearce EN. Thyroid hormone and obesity. Carr Opin Endocrinol Diabetes Obes 2012; 19:408-13.

19. Pacifico $\mathrm{L}$, Anania $\mathrm{C}$, Ferraro $\mathrm{F}$ et al. Thyroid function in childhood obesity and metabolic co-morbidity. Clin Chim Acta 2012; 413:396-405

20. Yiting $L$, Wei $W$, Xiasong $Y$ et al. Thyroid function and risk of non-alcoholic fatty liver disease in euthyroid subjects. Annals of Hepatology J 2018; 17(5):779-88.

21. Arafat $\mathrm{K}$, Farag $\mathrm{K}$, Mokhtar RR et al. Association and impact of non-alcoholic fatty liver disease on thyroid function. Int J Curr Res Sci 2017; 3(7):94-107.

22. Weiwei $H$, Xiaofei $A$, Ling $L$ et al. Relationship between Hypothyroidism and Non-alcoholic fatty liver disease: A systematic review and meta-analysis. Front Endocrinol 2017; 8:335.

23. Mazo D, Lima V, Stefano J et al. Gluco-lipidic indices in treated hypothyroidism associated with non-alcoholic fatty liver diseases. Dig Dis Sci 2012; 57:528-34.

24. Eshraghian A, Dabbaghmanesh $\mathrm{M}$, Eshraghian $\mathrm{H}$ et al. Nonalcoholic fatty liver disease in a cluster of Iranian population: thyroid status \& metabolic risk factors. Arch Iran Med 2013; 16:584-9.

25. Bril F, Lomonaco R, Orsak B et al. Relationship between disease severity, hyperinsulinemia and impaired insulin clearance in patients with non-alcoholic steatohepatitis. Hepatology 2014; 59:2178-87. 